\title{
Effects of Adding Behavioral Treatment to Opioid Detoxification With Buprenorphine
}

\author{
Warren K. Bickel, Leslie Amass, Stephen T. Higgins, Gary J. Badger, and Rebecca A. Esch \\ University of Vermont
}

\begin{abstract}
This trial assessed whether behavioral treatment improves outcome during a 26-week outpatient opioid detoxification. Thirty-nine opioid-dependent adults were assigned randomly to a buprenorphine dose-taper combined with either behavioral or standard treatment. Behavioral treatment included (a) a voucher incentive program for providing opioid-free urine samples and engaging in verifiable therapeutic activities and (b) the community reinforcement approach, a multicomponent behavioral treatment. Standard treatment included lifestyle counseling. Fifty-three percent of the patients receiving behavioral treatment completed treatment, versus $20 \%$ receiving standard treatment. The percentage of patients achieving $4,8,12$, and 16 weeks of continuous opioid abstinence were $68,47,26$, and 11 for the behavioral group and $55,15,5$, and 0 for the standard group, respectively. Behavioral treatment improved outcomes during outpatient detoxification.
\end{abstract}

Heroin and other opioid dependencies remain an important public health problem, and unfortunately, the prevalence of this problem appears to be escalating. For example, the number of young heroin users has recently increased, apparently because of the decreased price and increased purity of heroin (Hartnoll, 1994). In turn, more heroin and morphine-related emergency room visits have been reported (Swan, 1992). The criminal activity and intravenous (IV) drug use endemic to this population (Ball \& Ross, 1991) magnify the public health consequences of this trend. In particular, the IV route of administration increases the risk of contracting and spreading hepatitis and HIV infection (Curran, Jaffe, \& Hardy, 1988; Hser, Anglin, \& Power, 1993; Rice \& Kelman, 1989).

Not surprisingly, these developments have led to calls for the expansion of treatment services (McLellan, Arndt, Metzger, Woody, \& O'Brien, 1993). One treatment often available to opioid-dependent individuals, but rarely discussed or recommended, is outpatient detoxification with replacement medications such as methadone. This lack of interest may be well deserved given detoxification's documented lack of efficacy (Milby, 1988). Typically, studies of outpatient detoxification report high treatment

Warren K. Bickel and Stephen T. Higgins, Department of Psychiatry and Department of Psychology, University of Vermont; Leslie Amass and Rebecca A. Esch, Department of Psychiatry, University of Vermont; Gary J. Badger, Department of Medical Biostatistics, University of Vermont. Leslie Amass is now at the Department of Psychiatry, University of Colorado School of Medicine.

Preparation of this article was supported by Grants DA-06969 and 5T32 DA-07242 from the National Institute on Drug Abuse. The contributions of Alan Budney, John Brooklyn, Charles Frattini, Marlis Sorge, Hans Himmelein, John P. Crean, Florian Foerg, John Hughes, John Hrynyszyn, Marsha Dunham, David McGarry, Susan A. C. Griggs, Eric Jacobs, Nancy Petry, Ken Silverman, and Evan Tzanis are gratefully acknowledged.

Correspondence concerning this article should be addressed to Warren K. Bickel, Human Behavioral Pharmacology Laboratory, Department of Psychiatry, 38 Fletcher Place, Ira Allen School, Burlington, Vermont 05401 . retention and opioid abstinence when the dose of methadone is high but dramatically decreased retention and increased opioid use when the dose is tapered (Milby, 1988).

Similar outcomes have been reported with buprenorphine, a partial mu-opioid agonist currently being investigated as a replacement medication for opioid dependence (see Bickel \& Amass, 1995, for a review). For example, buprenorphine's effectiveness as a detoxification agent was compared with methadone in a random assignment outpatient detoxification study in which the medications were administered under double-blind and double-dummy conditions (Bickel et al., 1988). The active drug was tapered during the first 7 weeks, followed by placebo for 6 weeks. Retention was poor, with only $35 \%$ and $26 \%$ of the buprenorphine and methadone groups, respectively, remaining by the eighth week of treatment (the first week of placebo administration). Over $60 \%$ of the urine samples from both groups were opioid positive by the sixth week.

These poor outcomes raise an important question: Is the efficacy of outpatient opioid detoxification treatment strictly a function of the pharmacotherapy, or can efficacy be improved by combining pharmacotherapy with other nonpharmacological (i.e., psychosocial) interventions? The answer to this question could address the public health policy issue of whether to provide outpatient ambulatory detoxification treatment.

A similar question was addressed recently by a study examining methadone maintenance, a pharmacotherapy with documented efficacy. Methadone maintenance provides a constant daily dose of methadone indefinitely (see Ball \& Ross, 1991). Specifically, McLellan and colleagues examined the effects of three levels of psychotherapeutic services during methadone maintenance (McLellan et al., 1993). Minimum treatment provided daily methadone alone. Standard treatment consisted of methadone and counseling services. Enhanced treatment consisted of the same methadone and counseling services as provided by standard treatment, with the addition of employment and family counseling and on-site medical and psychiatric services. Sixty-nine percent, $41 \%$, and $19 \%$ of the methadone patients assigned to minimum, standard, and enhanced treatment 
services, respectively, engaged in persistent use of opioids and cocaine. Thus, a pharmacotherapy such as methadone can produce results ranging from poor to superior, depending on the additional psychosocial services provided.

Additional treatment services may also improve detoxification outcomes. Augmenting detoxification treatments with psychotherapeutic services might support the viability of conducting dose tapers or at least lead to research that identifies the type and number of supplemental services necessary to improve outcome. If supplemental services fail to influence outcome, then the policy of providing this treatment could be questioned.

A prior pilot study of opioid detoxification in our research clinic (Amass, Bickel, Higgins, \& Hughes, 1994) supported the application of the behavioral treatment approach developed by Higgins and colleagues (Higgins, Budney, Bickel, Foerg, et al., 1994; Higgins, Budney, Bickel, Hughes, et al., 1993; Higgins, Delaney, et al., 1991). This behavioral treatment of cocaine dependence consisted of two components. The first component was designed to positively reinforce cocaine abstinence by providing incentives for the submission of urine samples free of cocaine metabolites. The second component was designed to promote nondrug sources of reinforcement by improving employment status, family and social relations, and recreational activities. This treatment approach has demonstrated efficacy for cocaine dependence in controlled clinical trials and was readily adapted to opioid detoxification treatments (Amass, Bickel, Higgins, \& Hughes, 1994; Higgins, Budney, Bickel, Foerg, et al., 1994; Higgins, Budney, Bickel, Hughes, et al., 1993; Higgins, Delaney, et al., 1991).

In this article, we report the results of a randomized controlled trial comparing this behavioral treatment to standard treatment services during a 26-week outpatient detoxification with buprenorphine.

\section{Method}

\section{Participants}

Thirty-nine opioid-dependent patients ( 25 male, 14 female) with a mean age of 34.1 years (range $=19-45$ ) participated in this 26-week outpatient study (see Table 1 for patient characteristics). Patients were recruited over a 16-month period by means of newspaper, radio, and television advertisements and by word of mouth. All patients (a) met criteria for opioid dependence in accordance with the Diagnostic and Statistical Manual of Mental Disorders (3rd ed., revised; DSM-III-R; American Psychiatric Association, 1987); (b) met Food and Drug Administration (FDA) guidelines for methadone treatment (i.e., a history of opioid dependence and either significant current opioid use, e.g., opioid-positive urine samples, or signs of opioid withdrawal [i.e., gooseflesh, sweating, lacrimation, excessive yawning]); (c) were 18 years or older; (d) were free of psychosis or dementia or major medical disorders that would interfere with the administration of buprenorphine; and (e) were not pregnant as indicated by weekly negative urine tests (pregnancy tests were negative for all women throughout the study). All patients provided written informed consent after receiving a full explanation of the research and before study participation.

We determined a diagnosis of opioid dependence by the administration of the psychoactive substance abuse disorder sections of the DSM-III$R$ Criteria Checklist (Hudziak et al., 1993). This structured interview provides a set of questions to be read for each diagnosis. We used the $D S M-I I I-R$ criteria for opioid dependence to determine the diagnosis. The validity and reliability of this instrument have been established (Hudziak et al., 1996). DSM criteria were confirmed by a psychologist
Table 1

Participant Characteristics

\begin{tabular}{|c|c|c|}
\hline \multirow[b]{2}{*}{ Characteristic } & \multicolumn{2}{|c|}{$\%$ or $M \pm \mathrm{SD}$} \\
\hline & $\begin{array}{l}\text { Behavioral group } \\
\qquad(N=19)\end{array}$ & $\begin{array}{l}\text { Standard group } \\
(N=20)\end{array}$ \\
\hline \multicolumn{3}{|l|}{ Demographics } \\
\hline White & 100 & 95 \\
\hline Male & 63 & 65 \\
\hline Never married & 42 & 40 \\
\hline High school education & 95 & 85 \\
\hline Employed & 37 & 45 \\
\hline Age (in years) & $33.6 \pm 7.3$ & $34.6 \pm 8.0$ \\
\hline Weekly income (in dollars) & $221.6 \pm 231.0$ & $221.9 \pm 93.0$ \\
\hline \multicolumn{3}{|l|}{ Opioid use } \\
\hline Prior treatment & 79 & 80 \\
\hline Years of regular use & $8.8 \pm 7.1$ & $11.4 \pm 7.4$ \\
\hline Age of first use & $20.4 \pm 4.7$ & $21.0 \pm 3.9$ \\
\hline \multirow{2}{*}{\multicolumn{3}{|c|}{ Preferred route }} \\
\hline & & \\
\hline Intravenous & 63 & 65 \\
\hline Oral & 21 & 20 \\
\hline Intranasal & 16 & 15 \\
\hline \multicolumn{3}{|l|}{ Other drug dependence } \\
\hline Alcohol & 32 & 26 \\
\hline Cocaine & 26 & 35 \\
\hline Sedative & 26 & 32 \\
\hline Cannabis & 11 & 5 \\
\hline \multicolumn{3}{|l|}{ ASI composite scales } \\
\hline Medical & $0.32 \pm 0.41$ & $0.43 \pm 0.40$ \\
\hline Employment & $0.56 \pm 0.29$ & $0.56 \pm 0.33$ \\
\hline Alcohol & $0.14 \pm 0.24$ & $0.20 \pm 0.23$ \\
\hline Drug & $0.35 \pm 0.11$ & $0.41 \pm 0.10$ \\
\hline Psychiatric & $0.26 \pm 0.25$ & $0.36 \pm 0.29$ \\
\hline Legal & $0.26 \pm 0.31$ & $0.18 \pm 0.20$ \\
\hline Family-social & $0.23 \pm 0.21$ & $0.34 \pm 0.26$ \\
\hline Beck Depression Inventory & $23.7 \pm 12.0$ & $20.7 \pm 12.5$ \\
\hline \multicolumn{3}{|l|}{ Michigan Alcoholism } \\
\hline Screening Test & $19.0 \pm 15.6$ & $15.2 \pm 16.3$ \\
\hline
\end{tabular}

Note. All $p s \geq .10$ were based on $t$ tests for continuous measures and chi-square tests for categorical measures. ASI = Addiction Severity Index.

who also interviewed each patient. Other interviews and questionnaires that were administered included the Addiction Severity Index (McLellan, Luborsky, et al., 1985), the Beck Depression Inventory (Beck et al., 1961 ) and the Michigan Alcoholism Screening Test (Selzer, Vinokur, \& Van Rooijan, 1975). We developed additional questionnaires that were also completed and that provided information regarding demographic characteristics, drug history, and medical history.

Patients were assigned randomly at the end of the first week of treatment to one of the two treatments using minimum likelihood allocation (Aickin, 1982). This method of randomization is designed to achieve balance between treatment groups on patient characteristics likely to influence treatment outcome. Five characteristics were used to stratify patients to one of the two treatments: buprenorphine stabilization dose (i.e., 2,4 , or $8 \mathrm{mg} / 70 \mathrm{~kg}$ ), history of IV drug use during the past year, availability of a significant other who did not use drugs, gender, and prior history of buprenorphine treatment ( 10 patients participated in a buprenorphine clinical pharmacology study before this study). This method of group assignment successfully allocated patients to the behavioral or standard treatment groups without any significant differences ( $p$ $\leq .05$ ) on any baseline intake characteristics (Table 1).

\section{Setting}

This study was conducted at the University of Vermont's Substance Abuse Treatment Center's (SATC) Opioid Treatment Program, a feder- 
ally funded program providing free outpatient adult drug abuse treatment. This setting was where all services were provided. All patients, regardless of treatment assignment, received AIDS prevention education in the first month of treatment.

\section{Urinalysis Procedures}

Urine specimens were collected under staff observation from all patients on Mondays, Wednesdays, and Fridays and screened immediately on-site with the enzyme-multiplied immunoassay technique (Syva Corp., San Jose, CA). All specimens were screened for methadone, opiates, and propoxyphene, with one randomly selected specimen per week also screened for barbiturates, benzodiazepines, benzoylecgonine, and cannabinoids. Each opioid-positive sample was rescreened, with any discrepancy resulting in a independent test with thin-layer chromatography (TLC) methodology. A total of six samples required TLC testing. The results of the TLC testing confirmed the original opioid-positive result in each case. Breath alcohol samples were analyzed at the time when urine specimens were collected. Breath alcohol levels had to be less than or equal to $.05 \mathrm{~g} / \mathrm{ml}$ of air to receive medication.

\section{Counselors and Treatment Fidelity}

Five master's-level counselors ( 3 women and 2 men) with 1 to 7 years of experience with opioid-dependent patients administered treatment. Because the two treatments were not philosophically or theoretically inconsistent, the same counselors delivered both treatments. Each counselor received a minimum of 4 weeks of training in providing both treatments.

Treatment fidelity was insured in three ways. First, the behavioral treatment was a manually driven treatment, and the standard treatment was protocol driven (each session was to be conducted in the same way and to address the same material). Second, the behavioral and standard treatments provided by the counselors were reviewed in weekly group supervision with two psychologists. The two treatment groups were reviewed separately. These supervision meetings reviewed for each patient the treatment procedures used in the prior week and those to be used in the following week. Third, we conducted chart reviews every month to further ensure treatment fidelity.

\section{Behavioral Treatment}

The behavioral treatment consisted of community reinforcement and contingency management approaches originally developed for outpatient treatment of cocaine dependence (Higgins, Budney, Bickel, Foerg, et al., 1994; Higgins, Budney, Bickel, Hughes, et al., 1993; Higgins, Delaney, et al., 1991).

The voucher system involved systematically reinforcing abstinence as indicated by urinalysis results. Staff informed patients of their urinalysis results immediately after testing. Specimens that were negative for opioids (opiates, propoxyphene, and methadone) earned points that were recorded on vouchers and given to patients. Each point was worth \$.125. The first negative specimen was worth 29 points at $\$ .125$ per point or $\$ 3.63$. Each subsequent consecutive negative specimen increased the value of the voucher by one point (e.g., 30 points for the second, 31 points for the third, etc.). As an additional incentive for continuous opioid abstinence, a $\$ 5$ bonus was provided to patients for each set of three consecutive negative samples. Continuous abstinence throughout the 23-week period (Weeks 2-24) during which these contingencies were imposed would result in a patient receiving vouchers equivalent to a total of $\$ 658.38$ or $\$ 4.11$ per day. Note that Week 1 of the 26-week program was used to adjust the buprenorphine dose, and vouchers were not available during that time. Also, in an attempt to wean patients off the voucher program, we did not put these contingencies into effect for the last 2 treatment weeks.

Patients never received money directly. Instead, the cash equivalent of the points earned by patients were used by staff members to buy material reinforcers requested by patients (e.g., fishing license, dinner at a restaurant, automobile parts, establish phone service). These material reinforcers could be obtained at any time during treatment and were selected by the patient with the counselor who retained veto power over any item deemed to be inconsistent with the treatment goals. For a patient to receive these purchases, however, the most recent urine specimen had to be opioid negative.

Submission of an opioid-positive urine sample, or failing to submit a scheduled specimen, reset the value of vouchers to the initial $\$ 3.63$ level. Submission of five consecutive opioid-negative specimens returned the value of the vouchers to the level obtained before the reset. Points, once earned, could not be lost.

The other component of the behavioral treatment was the community reinforcement approach. These procedures, described in detail by Higgins and his colleagues (Higgins, Budney, Bickel, Hughes, et al., 1993), were implemented in two to three 1-hr individual counseling sessions per week. During therapy sessions, patients were provided, when appropriate, with relationship and employment counseling, instruction on antecedents and consequences of their opioid use, and assistance in developing new or reinitiating old recreational activities.

Significant-other participation integrated the community reinforcement approach and contingency management procedures. Significant others received the results of each urinalysis test by telephone. If the specimen was opioid negative, the significant other engaged in positive behavior with the patient that had been agreed upon previously with the counselor (e.g., going to the movies, back rub, or making dinner). If the specimen was opioid positive, he or she refrained from the agreedupon activities and instead offered assistance in achieving abstinence.

The community reinforcement approach and contingency management procedures were also integrated in another way for this treatment. Specifically, voucher reinforcement contingencies were placed on engaging in three verified activities per week that were selected by the patient and deemed by the counselor to be consistent with current therapeutic goals (e.g., develop resume, attend classes to receive a general equivalency diploma, apply for employment, attend YMCA exercise program). To verify the activities, the patient provided a receipt from the specified agency or organization dated appropriately or the counselor called the establishment. Thus, in this behavioral treatment, both opioid abstinence and completion of therapeutically appropriate activities were reinforced.

The activity voucher system was implemented during treatment Weeks 2 through 24 and was independent of urinalysis results. Points were equivalent in value to the vouchers used in conjunction with urinalysis results, but the reinforcement contingencies were altered. Specifically, each completed activity was worth one third of the maximum number of points (including bonuses) available weekly. All three activities had to be completed each week to advance to the next week's level of earnings. If all activities were completed during the 23 weeks of the voucher system, a patient would have completed 69 activities and received points worth $\$ 658.38$ or $\$ 4.11$ per day. Failure to complete an activity resulted in the patient's not receiving the scheduled points and prevented advancement to the next week's level of earnings until the required activities were completed.

\section{Standard Methadone-Style Counseling}

The standard treatment was based on Ball and Ross's (1991) survey of six methadone clinics. This survey assessed the duration and content of counseling across these clinics. For the present trial, individual counseling sessions were held once per week for a duration of $37 \mathrm{~min}$. The content of each session was to address compliance with the program rules (one third of session) and rehabilitation (two thirds of session). When addressing rehabilitation, counselors would suggest or devise plans to decrease drug use, seek employment, or improve the patients' living arrangements. The urine collection schedule was the same as that for the behavioral treatment, but urinalysis results were not used in any 
voucher or reinforcement system. Counselors were informed of urinalysis results and discussed them with patients.

\section{Medication Administration}

The detoxification lasted 160 days for patients regardless of dose. Daily clinic attendance was required to receive medication. After the first week (described later), patients were maintained for an additional 72,42 , or 7 days for the 2,4 , or $8 \mathrm{mg} / 70 \mathrm{~kg}$ dose, respectively. During the dose reduction phase, the dose was decreased gradually approximately $10 \%$ every 5 days for the remainder of the 160 days. Thus, the completion of the dose reduction took $2.8,3.8$, and 4.8 months for the 2 , 4 , and $8 \mathrm{mg} / 70 \mathrm{~kg}$ dose conditions, respectively. This combination of dose stabilization and reduction schedule ensured that patients, regardless of assigned dose, received the same number of drug administrations. Moreover, for doses that overlapped across the three dose conditions, patients received the same dose reduction at the same day in the study. At the completion of the dose-reduction phase, placebo was administered for an additional 3 weeks.

The stabilization dose was established during the first week of participation before treatment assignment. Patients were initially placed on a $4 \mathrm{mg} / 70 \mathrm{~kg}$ dose of buprenorphine. The dose was increased to $8 \mathrm{mg} /$ $70 \mathrm{~kg}$ for patients who exhibited withdrawal signs and symptoms. The dose was decreased to $2 \mathrm{mg} / 70 \mathrm{~kg}$ for patients who exhibited opioid intoxication. Exceptions to this procedure occurred when patients reported either low or high levels of opioid use. Patients reporting low levels were placed directly on the $2 \mathrm{mg} / 70 \mathrm{~kg}$ dose, whereas patients who reported high levels were started directly on the $8 \mathrm{mg} / 70 \mathrm{~kg}$ dose using a 3-day rapid induction procedure (Johnson, Cone, Henningfield, \& Fudala, 1989). Eleven, 19, and 9 patients were placed on the 2,4 , and $8 \mathrm{mg} / 70 \mathrm{~kg}$ maintenance dose, respectively.

Buprenorphine hydrochloride was prepared as a stock concentration of $8 \mathrm{mg} / \mathrm{ml}$ in $35 \%$ ethanol ( $\mathrm{vol} / \mathrm{vol}$ ) for sublingual administration. Buprenorphine placebo consisted of sublingual ethanol solution alone. The volume (in milliliters) of each patient's dose remained constant within patients. Patients held the medication under their tongue for 10 min without speaking. Medications were administered with a Ped-Pod Oral Dispenser (SoloPak Laboratories; Franklin Park, IL) under doubleblind conditions.

\section{Outcome Measures}

Treatment retention. Retention was defined as the percentage of participants in each treatment retained in treatment at the end of each of the 26 treatment weeks, beginning with the first day of treatment. Participants who missed 3 consecutive days of medication or failed to provide five consecutive urine samples were discharged from treatment. For both treatments, the counselor made a telephone call on the second day of missed medication or when the fourth consecutive urine sample was missed to encourage the patient to return to the clinic.

Opioid abstinence. One week of continuous opioid abstinence was defined as three consecutive opioid-negative urine specimens. The longest period of continuous abstinence achieved was compiled for each participant.

Activities completed. Each activity was scored as either completed or not completed on the basis of verification (see "Behavioral Treatment" section, for verification procedures). Three activities were assigned per week for 23 weeks in the behavioral treatment.

\section{Results}

Chi-square tests were used to compare treatment groups on dichotomous outcome measures such as percentage of patients retained through study completion and percentage of patients achieving at least $4,8,12$, and 16 weeks of continuous absti- nence. Survival distributions associated with weeks retained in treatment were compared across treatment groups using a logrank test. Missing urine specimens were treated as opioid positive as recommended in substance abuse treatment research ( $\mathrm{Na}$ than \& Lansky, 1978). Correlation analysis was used to examine the relationship between activities completed and outcome measures associated with abstinence and retention within the behavioral treatment group. Statistical significance was determined at $p \leq .05$.

\section{Patient Retention}

A chi-square test indicated that significantly more patients receiving behavioral treatment $(53 \%)$ completed the 26 -week study (end of buprenorphine plus three weeks of placebo) than patients receiving standard treatment $(20 \%), \chi^{2}(1, N=39)=$ $4.5, p=.03$ (see Figure 1). The survival distributions associated with treatment retention were not statistically different from one another based on a log-rank test, $\chi^{2}(1, N=39)=2.9, p=$ .09 (Figure 1).

\section{Opioid Abstinence}

Significantly more participants in the behavioral treatment group achieved at least 8 weeks of continuous abstinence, $\chi^{2}(1$, $N=39$ ) $=4.8, p=.03$, compared with the standard group ( $47 \%$ vs. $15 \%$; Figure 2 ). A similar nonsignificant trend occurred for the percentage of participants in the behavioral treatment group who achieved at least 12 weeks of abstinence, $\chi^{2}(1, N=39)$ $=3.4, p=.06$, compared with the standard group ( $26 \%$ vs. $5 \%$ ). The behavioral group also achieved greater abstinence than the standard treatment groups on every other abstinence measure, although the difference between the groups did not reach statistical significance. For example $68 \%$ and $11 \%$ of the behavioral treatment group achieved 4 and 16 weeks of continuous abstinence, respectively, whereas $55 \%$ and $0 \%$ of the standard treatment group achieved continuous abstinence for those same respective durations. The percentage of patients who were opioid negative at the end of buprenorphine administration, and 3 weeks later at the end of placebo administration, were $32 \%$ and $21 \%$ for the behavioral group, respectively, and $15 \%$ and $10 \%$ for the standard group, respectively.

There was no significant difference between the two groups regarding the percentage of patients who were positive during the trial for barbiturates, benzodiazepines, cocaine, and THC or the frequency of use of those that used these substances (Table 2).

\section{Relation to Activities Completed and Treatment Outcome During Behavioral Treatment}

The total number of activities completed and weeks of continuous opioid abstinence achieved correlated, $r=.76, p<.001$, in the behavioral treatment group. A positive correlation was observed between the number of activities completed and weeks retained in treatment, $r=.88, p<.001$.

\section{Cost of Behavioral Treatment}

The mean worth of vouchers earned per patient was $\$ 274$ $(S D=\$ 197)$ for the urinalysis system and $\$ 305(S D=\$ 234)$ for the activity system. The mean worth of vouchers earned by 


\section{Treatment Retention}

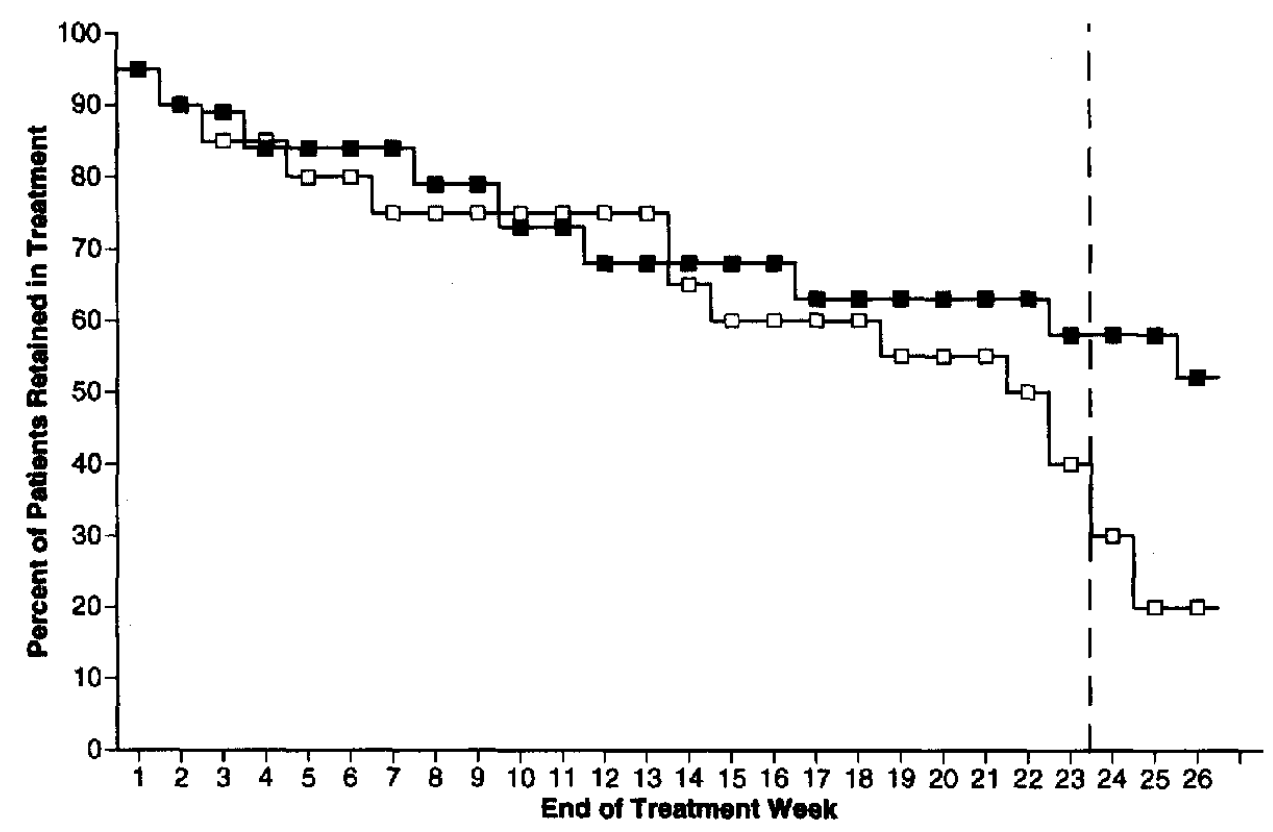

Figure 1. Percentage of patients retained in treatment at the end of each treatment week. The dashed vertical line before Week 24 indicates the start of the placebo period as a function of treatment week. Closed squares indicate behavioral treatment; open squares indicate standard treatment.

patients across both systems was $\$ 579(S D=\$ 416)$. Dividing the mean amount of money earned by the mean number of weeks patients were retained results in a daily cost for the contingency management program of $\$ 4.32$ per participant.

\section{Discussion}

This study demonstrates that a combined behavioral-pharmacological treatment package can augment treatment outcomes

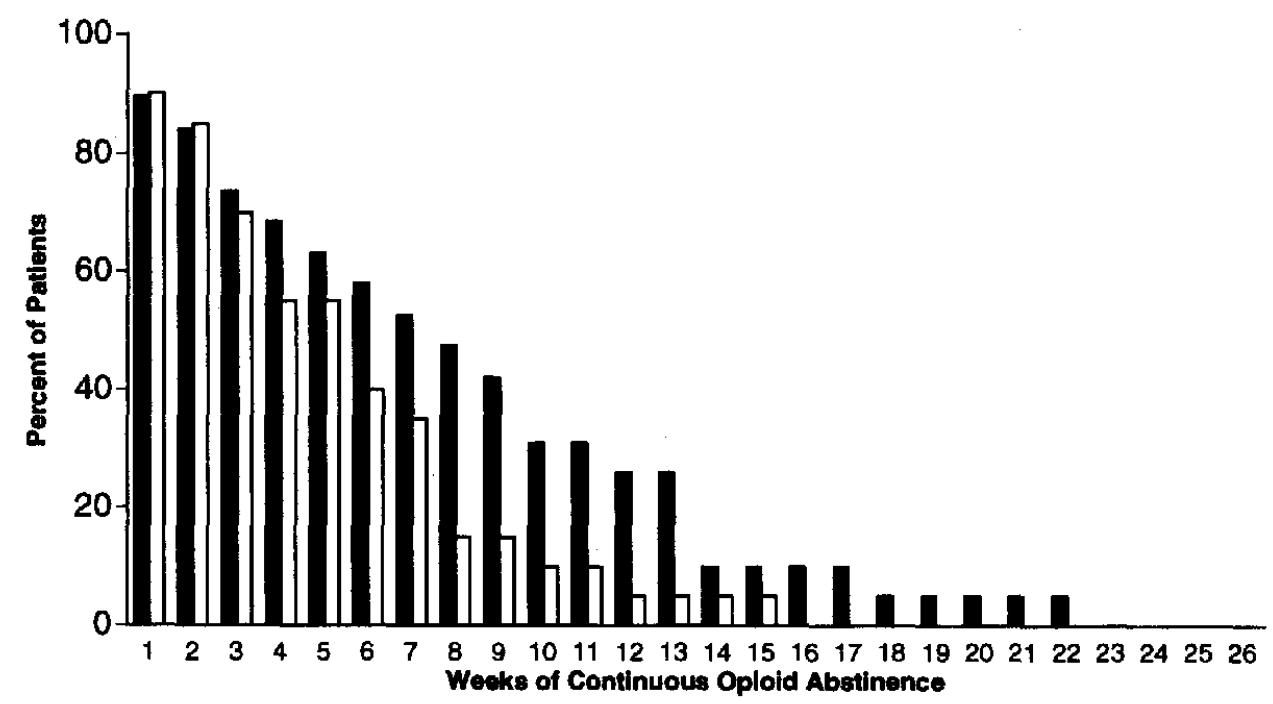

Figure 2. Distribution of documented continuous opioid abstinence observed in each treatment group. The height of each bar represents the percentage of patients achieving a duration of continuous abstinence greater than or equal to the number of weeks indicated. Note that the weeks of continuous opioid abstinence could occur anywhere within the 26-week study. Closed squares indicate behavioral treatment; open squares indicate standard treatment. 
Table 2

Other Drug Use

\begin{tabular}{|c|c|c|c|c|c|c|}
\hline \multirow[b]{2}{*}{ Drug } & \multicolumn{3}{|c|}{$n$ (and $\%$ ) of participants positive ${ }^{a}$} & \multicolumn{3}{|c|}{$M \pm S D$ for $\%$ specimens positive $\mathrm{b}^{\mathrm{b}}$} \\
\hline & $\begin{array}{c}\text { Behavioral } \\
\text { treatment }\end{array}$ & $\begin{array}{l}\text { Standard } \\
\text { treatment }\end{array}$ & $p^{\mathrm{c}}$ & $\begin{array}{c}\text { Behavioral } \\
\text { treatment }\end{array}$ & $\begin{array}{l}\text { Standard } \\
\text { treatment }\end{array}$ & $p^{\mathrm{c}}$ \\
\hline Barbiturates & $9(47)$ & $6(30)$ & .26 & $36 \pm 13$ & $22 \pm 16$ & .51 \\
\hline Benzodiazepines & $17(89)$ & $15(75)$ & .24 & $55 \pm 8$ & $58 \pm 10$ & .82 \\
\hline Cannabinoids & $9(47)$ & $11(55)$ & .63 & $45 \pm 11$ & $40 \pm 9$ & .72 \\
\hline Cocaine & $12(63)$ & $11(55)$ & .61 & $19 \pm 4$ & $39 \pm 11$ & .15 \\
\hline
\end{tabular}

Note. Behavioral treatment $n=19 ;$ standard treatment $n=20$.

a Participants were defined as positive if at least one positive specimen was obtained during the trial. ${ }^{b}$ This only includes participants who were positive for substance use during the trial. ${ }^{\mathrm{c}}$ Chi-square test. ${ }^{\mathrm{d}} t$ test.

during ambulatory opioid detoxification. Although the magnitude of that effect was modest, it suggests that detoxification outcomes can be modified. Perhaps further additions to the treatment package will result in further improvement in detoxification outcome. We comment here on eight points related to these results.

First, the behavioral treatment improved patient retention. Over twice as many patients receiving behavioral treatment $(50 \%)$, compared with standard treatment ( $20 \%)$, were retained for 3 additional weeks of placebo administration beyond the last dose of buprenorphine. The retention in the behavioral treatment group exceeded that from a prior buprenorphine detoxification trial that retained approximately $35 \%$ of patients until the end of buprenorphine administration, and fewer than $5 \%$ were retained by the end of an additional 3 weeks of placebo (Bickel, Stitzer, et al., 1988). Enhanced retention appears to be a consistent result of behavioral treatments and is an important outcome measure, given the association between longer retention and improvements on other treatment outcomes (Higgins, Budney, Bickel, Foerg, et al., 1994).

Second, although several measures of abstinence did not achieve statistical significance, the group receiving behavioral treatment exhibited greater abstinence than the group receiving standard treatment. Importantly, significantly more participants achieved extended periods of continuous opioid abstinence (e.g., 8 weeks) in the behavioral than in the standard treatment group. Of course, the abstinence, as well as the retention results, may have to be improved further to be accepted as a useful treatment approach. Nonetheless, they demonstrate that detoxification results are not inherently poor. A related issue was the specificity of the results; that is, the effects of the behavioral treatment on opioid use were not reflected in urinalysis results for other drugs. This specificity of effect is consistent with other applications of contingency management procedures (e.g., Higgins, Budney, Bickel, Foerg, et al., 1994) and suggests that obtaining decreases in other drug use requires that these drugs be added to the imposed contingencies (Budney, Higgins, Delaney, Kent, \& Bickel, 1991).

Third, the strong relationship between the completion of activities and both opioid abstinence and retention underscores the importance of competing sources of nondrug reinforcement in suppressing drug use (Bickel \& DeGrandpre, 1996; Vuchinich \& Tucker, 1988). Moreover, the present study suggests a continuous relationship where greater completion of altemative activities was related to more improved treatment outcomes. These results are consistent with the findings of prior basic human and animal research that demonstrates suppression of drug taking by making competing sources of nondrug reinforcement available (Bickel, DeGrandpre, Higgins, Hughes, \& Badger, 1995; Carroll, Carmona, \& May, 1991; Higgins, Bickel, \& Hughes, 1994; Nader \& Woolverton, 1992). Additional clinical support comes from the positive treatment outcomes that resulted from the application of the community reinforcement approach by itself (Sisson \& Azrin, 1989). Of course, this relationship may not be causal, and it is open, therefore, to other interpretations. For example, patients may vary in their compliance, and the more compliant they are, the more they will engage in all aspects of treatment including abstinence, retention, and engagement in specified activities. Future prospective research should examine whether engaging in these activities in and of themselves contributes to improved treatment efficacy.

Fourth, the results of the present trial replicate previous research by Higgins and his colleagues (Higgins, Budney, Bickel, Foerg, et al., 1994; Higgins, Budney, Bickel, Hughes, et al., 1993; Higgins, Delaney, et al., 1991). Higgins et al. demonstrated that their multicomponent behavioral treatment improves retention, and abstinence outcomes relative to a standard treatment in cocaine-dependent patients (Higgins, Budney, Bickel, Hughes, et al., 1993; Higgins, Delaney, et al., 1991). Other research has successfully extended elements of that treatment to cocaine-dependent methadone maintenance patients (Silverman et al., 1996). This study further extends the effectiveness of this treatment to opioid-dependent individuals receiving outpatient buprenorphine detoxification. Of course, there were numerous differences between the treatments in the present trial, such as total contact time and the contingency management procedures, and the design does not permit any conclusion as to which treatment component was central to the outcomes observed.

Fifth, the present results replicate and extend prior research with methadone-maintained patients, which demonstrated that supplementing methadone maintenance with greater treatment services significantly improves treatment outcomes (McLellan et al., 1993). Future research with the combined behavioral and pharmacological treatment could also use incentives inherent to the pharmacotherapy itself. For example, prior research with methadone has shown it to function as a reinforcer (Bickel, Higgins, \& Stitzer, 1986), and it has been used in a variety of 
ways to improve outcomes in maintenance and detoxification treatments (Bickel et al., 1989; Higgins, Stitzer, Bigelow, \& Liebson, 1986; Stitzer, Bigelow, \& Liebson, 1979; Stitzer, Bickel, Bigelow, \& Liebson, 1986). Given that buprenorphine can also function as a reinforcer, it could be used in an analogous fashion to further improve outcomes of the behavioral treatment (see Bickel \& Amass, 1995). Moreover, buprenorphine's unique pharmacology may lead to novel applications in an incentive program (Amass, Bickel, Higgins, \& Badger, 1994).

Sixth, one limitation of the study is that the same therapists provided both treatments. This may be preferable to confounding treatments with counselor effects. Nonetheless, this design choice leads to the possibility that therapists may come to expect poorer outcomes with the standard treatment and behave in ways consistent with that expectation. Such an expectation should result in poorer outcomes with the control group than would have otherwise been expected. The methods used to ensure treatment fidelity should minimize this possibility. However, to explore this further, one may compare the results of the control group from the present study with the results from the prior random assignment studies of opioid detoxification. For example, Higgins et al. (1986) examined the effects of contingent methadone delivery on drug-free urine samples during a 13week detoxification procedure ( 10 weeks to complete the dose reduction followed by 3 weeks of placebo). The control group was retained for an average of 64 days in their 90-day (13week) detoxification schedule, which resulted in retention for $71 \%$ of the study duration. Another study, described earlier, compared buprenorphine and methadone detoxification treatments (Bickel et al., 1988). Dose reductions for both drugs were completed over 7 weeks followed by 6 weeks of placebo. By the end of the third week of placebo administration, patients were retained for $69 \%$ and $57 \%$ of the study duration (dose reduction and 3 weeks of placebo) for the buprenorphine and methadone groups, respectively. In the present trial, retention in the control group was a mean of 17.4 weeks of the 26 weeks (23 weeks of buprenorphine medication and 3 weeks of placebo), which resulted in a retention for $67 \%$ of the study duration. Thus, the results of the control group from the present study produced results that were comparable with other controlled random assignment trials examining opioid detoxification treatments and suggests that this weakness may have not been influential.

Seventh, one limitation of this study is the limited power that was due to the small number of patients: The small number rendered the detection of differences between the groups a difficult task. Moreover, differences between the treatment groups may have been obscured by the efficacy of buprenorphine itself. This is supported by the greater likelihood of detecting treatment effects toward the end of the detoxification period. Thus, a larger number of patients may need to be enrolled to detect significant differences on more measures when such treatments are combined with an effective pharmacotherapy.

Eighth, another potential limitation of the present study is limited generalizability as a result of patient characteristics. The primary difference between the sample in the present study and other patients receiving treatment for opioid dependence is the racial make-up of the patients. In Vermont, fewer non-White patients exist than in urban areas. Otherwise, the characteristics outlined in Table 1 are similar to those reported in Ball and
Ross's survey of six methadone clinics and those reported in McLellan's recent study on methadone patients (Ball \& Ross, 1991; McLellan et al., 1993). Although racial or other unmeasured differences in patient characteristics may limit the generalizability of the present report, the behavioral treatment studied here has been replicated successfully in racially diverse urban populations receiving methadone maintenance therapy (Silverman et al., 1996).

In conclusion, the pursuit of combined behavioral and pharmacological interventions is important for the development of a comprehensive approach to drug abuse treatment. Evidence from the present and prior studies demonstrates that a pharmacotherapy's efficacy for maintenance and detoxification services can be rendered better or worse depending on the additional psychosocial services provided (McLellan et al., 1993). Although outcomes after detoxification may still not be optimal, the behavioral treatment examined here demonstrates that detoxification results are malleable and supports the possibility of developing opioid detoxification as an efficacious treatment.

\section{References}

Aickin, M. (1982). A program for balancing the allocation of patients in a clinical trial. Computers and Biomedical Research, 15, 519-524.

Amass, L., Bickel, W. K., Higgins, S. T., \& Badger, G. J. (1994) . Alternate-day dosing during buprenorphine treatment of opioid dependence. Life Sciences, 54, 1215-1228.

Amass, L., Bickel, W. K., Higgins, S. T., \& Hughes, J. R. (1994). A preliminary investigation of outcome following gradual or rapid buprenorphine detoxification. Journal of Addictive Diseases, 13, 3547.

American Psychiatric Association. (1987). Diagnostic and statistical manual of mental disorders (3rd ed., rev.). Washington, DC: Author.

Ball, J. C., \& Ross, A. (1991). The effectiveness of methadone maintenance treatment. New York: Springer-Verlag.

Beck, A. T., Ward, C. H., Mendelson, M., Mock, J., \& Erbaugh, J. (1961). An inventory for measuring depression. Archives of General Psychiatry, 4, 561-571.

Bickel, W. K., \& Amass, L. (1995). Buprenorphine treatment of opioid dependence: A review. Experimental and Clinical Psychopharmacol. ogy, 3, 477-489.

Bickel, W. K., \& DeGrandpre, R. J. ( 1996). Basic psychological science speaks to drug policy: Drug cost and competing reinforcement. In W. K. Bickel \& R. J. DeGrandpre (Eds.), Drug policy and human nature: Psychological perspectives on the control, prevention, and treatment of illicit drug use (pp. 31-52). New York: Plenum Press.

Bickel, W. K., DeGrandpre, R. J., Higgins, S. T., Hughes, J. R., \& Badger, G. J. (1995). Effects of simulated employment and recreation on drug taking: A behavioral economic analysis. Experimental and Clinical Psychopharmacology, 3, 467-476.

Bickel, W. K., Higgins, S. T., \& Stitzer, M. L. (1986). Choice of blind methadone dose increases by methadone maintenance patients. Drug and Alcohol Dependence, 18, 165-171.

Bickel, W. K., Rizzuto, P., Zielony, R. D., Klobas, J., Panagiosonlis, P., Mernit, R., \& Knight, W. F. (1989). Combined behavioral and pharmacological treatment of alcoholic methadone patients. Journal of Substance Abuse, 1, 161-167.

Bickel, W. K., Stitzer, M. L., Bigelow, G. E., Liebson, I. A., Jasinski, D. R., \& Johnson, R. E. (1988). A clinical trial of buprenorphine: Comparison with methadone in the detoxification of heroin addicts. Clinical Pharmacology and Therapeutics, 43, 72-78.

Budney, A. J., Higgins, S. T., Delaney, D. D., Kent, L., \& Bickel, W. K. (1991). Contingent reinforcement of drug-free urine samples with 
individuals abusing both cocaine and marijuana. Journal of Applied Behavioral Analysis, 24, 657-665.

Carroll, M. E., Carmona, G. G., \& May, S. A. (1991). Modifying drugreinforced behavior by altering the economic conditions of the drug and nondrug reinforcer. Journal of Experimental Analysis of Behavior, 56, 361-376.

Curran, J. W., Jaffe, H. W., \& Hardy, A. M. (1988). Epidemiology of HIV infection and AIDS in the United States. Science, 33, 15-26.

Hartnoll, R. L. (1994). Opiates: Prevalence and demographic factors. Addiction, 89, 1377-1383.

Higgins, S. T., Bickel, W. K., \& Hughes, J. R. (1994). Influence of an alternative reinforcer on human cacaine self-administration. Life Science, 55, 179-187.

Higgins, S. T., Budney, A. J., Bickel, W. K., Foerg, F. E., Donham, R., \& Badger, G. J. (1994). Incentives improve outcome in outpatient behavioral treatment of cocaine dependence. Archives of General Psychiatry, 51, 568-576.

Higgins, S. T, Budney, A. J., Bickel, W. K., Hughes, J. R., Foerg, F., \& Badger, G. (1993). Achieving cocaine abstinence with a behavioral approach. American Journal of Psychiatry, 150, 763-769.

Higgins, S. T., Delaney, D. D., Budney, A. J., Bickel, W. K., Hughes, J. R., Foerg, F., \& Fenwick, J. (1991). A behavioral approach to achieving initial cocaine abstinence. American Journal of Psychiatry, 148, 1218-1224.

Higgins, S. T., Stitzer, M. L., Bigelow, G. E., \& Liebson, I. A. (1986). Contingent methadone delivery: Effects on illicit-opiate use. Drug and Alcohol Dependence, 17, 311-322.

Hser, Y., Anglin, M. D., \& Power, K. (1993). A 24-year follow-up of California narcotic addicts. Archives of General Psychiatry, 50, 577584.

Hudziak, J., Helzer, J. E., Wetzel, M. W., Kessel, K. B., McGee, B., Janca, A., \& Przybeck, P. (1993). The use of the DSM-III-R checklist for initial diagnostic assessments. Comprehensive Psychiatry, 34, $375-383$.

Hudziak, J. J., Jarvis, M. R., Przybeck, T. R., Schaffer, A., Janca, A., Hudgens, R. W. (1996). Validity and inter-rater reliability of nonclinician diagnosis with the DSM-III-R Checklist Interview. Manuscript under review.

Johnson, R. E., Cone, E. J., Henningfield, J. E., \& Fudala, P. J. ( 1989). Use of buprenorphine in the treatment of opioid addiction: I. Physiologic and behavioral effects during a rapid dose induction. Clinical Pharmacology and Therapeutics, 46, 335-343.

McLellan, A. T., Arndt, I. O., Metzger, D. S., Woody, G. E., \& O'Brien, C. P. (1993). The effects of psychosocial services in substance abuse treatment. Journal of the American Medical Association, 269, 19531959.

McLellan, A. T., Luborsky, L., Cacciola, J., Griffith, J., Evans, F., Barr, H. L., \& O'Brien, C. P. (1985). New data from the Addiction Severity Index: Reliability and validity in three centers. Journal of Nervous and Mental Diseases, 173, 412-423.

Milby, J. B. (1988). Methadone maintenance to abstinence. How many make it? Journal of Nervous and Mental Diseases, 176, 409-422.

Nader, M. A., \& Woolverton, W. L. (1992). Choice between cocaine and food by rhesus monkeys: Effect of conditions of food availability. Behavioral Pharmacology, 3, 635-638.

Nathan, P. E., \& Lansky, D. (1978). Common methodological problems in research on the addictions. Journal of Consulting and Clinical Psychopharmacology, 46, 713-726.

Rice, D. P., \& Kelman, S. (1989). Measuring comorbidity and overlap in the hospitalization cost for alcohol and drug abuse and mental illness. Inquiry, 26, 249-260.

Selzer, M. L., Vinokur, A., \& Van Rooijan, L. (1975). A self-administered short Michigan Alcoholism Screening Test (SMAST). Journal of Studies of Alcohol, 36, 117-126.

Silverman, K., Higgins, S. T., Brooner, R. K., Montoya, I. D., Cone, E. J., Schuster, C. R., \& Preston, K. L. (1996). Sustained cocaine abstinence in methadone maintenance patients through voucher-based reinforcement therapy. Archives of General Psychiatry, 53, 409-415.

Sisson, R. W., \& Azrin, N. H. (1989). The community reinforcement approach. In R. K. Hester \& W. R. Miller (Eds.), Handbook of alcoholism treatment approaches: Effective alternatives (pp. 242-258). New York: Pergamon Press.

Stitzer, M. L., Bickel, W. K., Bigelow, G. E., \& Liebson, I. A. (1986). Effects of methadone dose contingencies on urinalysis test results of poly-abusing methadone-maintenance patients. Drug and Alcohol Dependence, 18, 341-348.

Stitzer, M. L., Bigelow, G. E., \& Liebson, I. A. (1979). Reinforcement of drug abstinence: I. A behavioral approach to drug abuse treatment. In N. K. Krasnegor (Ed.), Behavioral analysis and treatment of drug abuse (pp. 65-90). Washington, DC: U.S. Government Printing Office.

Swan, N. (1992). Heroin and cocaine-related visits to hospital emergency rooms continue to increase nationwide. NIDA Notes, 7, 9-10.

Vuchinich, R. E., \& Tucker, J. A. (1988). Contributions from behavioral theories of choice as a framework to an analysis of alcohol abuse. Journal of Abnormal Psychology, 92, 408-416.

Received March 26, 1996

Revision received September 19, 1996 Accepted January 30, 1997 